\title{
Subgroups of primary Sjögren's syndrome. Sjögren's syndrome in male and paediatric Greek patients
}

Alexandros A Drosos, Eleni K Tsiakou, Niki Tsifetaki, Eudokia N Politi, Antigoni Siamopoulou-Mavridou

\begin{abstract}
Objectives-To describe the clinical and serological findings in male and paediatric Sjögren's syndrome (SS) patients.

Patients and Methods-Using the European criteria for the diagnosis of SS 12 male and 13 paediatric patients were identified and compared with those of 30 consecutive unselected adult female SS patients.

Results-The mean (SD) age of paediatric patients was 9.4 (2.2) years, ranging from 6 to 14 years. Recurrent parotid gland enlargement was the initial clinical manifestation in the majority of the children with a statistical significance compared with male $(p<0.01)$ and with female patients $(p<0.0001)$. Sicca manifestations were the most common clinical symptoms in male and female patients at disease onset. The systemic manifestations were similar among the three groups except that men showed lower frequency of arthritis $(p<0.05)$ and Raynaud's phenomenon $(p<0.05)$ compared with women. No differences were found among the immunological profile of children and female patients, while male patients had a lower frequency of antinuclear antibodies $(p<0.025)$ and antibodies to $\operatorname{Ro}(\mathrm{SSA})$ nuclear antigens $(p<0.025)$ compared with women.

Conclusion-Primary SS is rare in children and men in Greece. Recurrent parotid gland enlargement is the most common clinical finding at disease onset in children. Male patients seem to have less systemic manifestations and lower frequency of autoantibodies.
\end{abstract}

(Ann Rheum Dis 1997;56:333-335)

Primary Sjögren's syndrome (SS) is a chronic autoimmune disorder affecting the lacrimal and salivary glands, as well as other exocrine glands leading to functional impairment and mucosal dryness. It is often associated with systemic extraglandular manifestations, and laboratory tests show B cell polyclonal hyperactivity. It affects predominantly women at middle age. ${ }^{12}$ Although the first case reported by Dr Mikulicz was a 42 year old man, ${ }^{3}$ there are only few reports describing the clinical picture of SS in males. ${ }^{4-6}$ In children only case reports of SS have been reported. ${ }^{7-10}$
In this study we present 12 male and 13 paediatric patients and compare their clinical and serological findings with those of 30 adult female SS patients.

\section{Patients and methods}

In the past 15 years we have diagnosed 16 children and 13 men with primary SS. We have prospectively reevaluated the exocrine glands and related symptomatology of all these patients, and compared them with those of 30 sequential, unselected adult female SS patients. None of our female paediatric patients had menses at the onset of the disease while 22 of 30 adult female SS patients had regular menstrual cycles during the study period.

All patients had a complete physical examination and routine laboratory tests. After written consent was obtained, all patients underwent a minor salivary gland biopsy under local anaesthesia. All patients' serum samples were tested for the presence of rheumatoid factor (RF) (latex test), antinuclear antibodies (ANA) (indirect immunofluorescence using Hep-2 cell lines), antibodies to Ro(SSA), $\mathrm{La}(\mathrm{SSB}), \mathrm{Sm}$ and $\mathrm{U}_{1} \mathrm{RNP}$ nuclear antigens (counter immunoelectrophoresis), and $\mathrm{C}_{3}$ and $\mathrm{C}_{4}$ complement values.

For the diagnosis of primary SS we used the preliminary European criteria. ${ }^{11}$

For statistical analysis of the results, the Student $t$ test and $\chi^{2}$ test with Yates's correction were used.

\section{Results}

We have identified 13 paediatric (11 girls, 2 boys) and 12 male patients. Paediatric and male SS patients were identified from our SS cohort of 292 primary SS cases and represent about $4 \%$ of our SS population, while the total of juvenile polyarthritis patients seen in our paediatric clinic was 298. Three paediatric patients and one man were excluded from the study because: two children had an associated disease, juvenile chronic polyarthritis and the other one did not meet the European criteria for SS. The male patient, who also was excluded from the study, had sarcoidosis.

Table 1 shows the mean age and disease duration. Recurrent parotid gland enlargement (RPGE) was the initial clinical manifestation in the majority of the children (77\%) with a statistical significance compared with other groups. Sicca manifestations were the most
Accepted for publication 14 February 1997 
Table 1 Comparison of clinical and laboratory findings between subgroups with primary Sjögren's syndrome patients

\begin{tabular}{|c|c|c|c|c|}
\hline Characteristics & Children $(n=13)$ & $\operatorname{Men}(n=12)$ & Female $(n=30)$ & p Value \\
\hline Age at onset $(y)($ mean (SD)) & $9.4(2.8)$ & $45.3(5.5)$ & $46.4(6.1)$ & \\
\hline Disease duration (y) (mean(SD)) & $6.5(3.2)$ & $11.2(5.0)$ & $10.5(5.4)$ & \\
\hline \multicolumn{5}{|l|}{ First manifestations } \\
\hline Sicca syndrome & $3(23 \%)$ & $9(75 \%)^{\star}$ & $23(77 \%) \dagger$ & $\mathrm{p}<0.05^{\star}, \mathrm{p}<0.005 \dagger$ \\
\hline RPGE & $10(77 \%)$ & $2(17 \%)^{\star}$ & $4(13 \%) \dagger$ & $\mathrm{p}<0.01^{\star}, \mathrm{p}<0.0001 \dagger$ \\
\hline EGM & - & $1(8 \%)$ & $3(10 \%)$ & NS \\
\hline \multicolumn{5}{|c|}{ Extraglandular manifestations during disease } \\
\hline Constitutional symptoms & $3(23 \%)$ & $4(33 \%)$ & $7(23 \%)$ & NS \\
\hline Musculoskeletal & $3(23 \%)$ & $1(8 \%)$ & $14(47 \%)$ & $\mathrm{p}<0.05$ \\
\hline Raynaud's phenomenon & $4(31 \%)$ & $1(8 \%)$ & $13(43 \%)$ & $\mathrm{p}<0.05$ \\
\hline Lymphadenopathy & $3(23 \%)$ & $4(33 \%)$ & $5(17 \%)$ & NS \\
\hline Hepatomegaly & $1(8 \%)$ & $2(17 \%)$ & $4(13 \%)$ & NS \\
\hline Skin involvement & - & - & $1(3 \%)$ & NS \\
\hline Pulmonary involvement & - & - & $1(3 \%)$ & NS \\
\hline \multicolumn{5}{|l|}{ Autoantibodies } \\
\hline $\mathrm{RF}$ & $6(46 \%)$ & $8(67 \%)$ & $16(53 \%)$ & NS \\
\hline ANA & $9(69 \%)$ & $4(33 \%)$ & $23(77 \%)$ & $\mathrm{p}<0.025$ \\
\hline Ro (SSA) & $7(54 \%)$ & $2(17 \%)$ & $19(63 \%)$ & $\mathrm{p}<0.025$ \\
\hline $\mathrm{La}(\mathrm{SSB})$ & $4(31 \%)$ & $1(8 \%)$ & $11(37 \%)$ & NS \\
\hline
\end{tabular}

and $\mathrm{men} . \dagger=$ statistically significant between children and women.

common clinical symptoms in male and female patients at disease onset. The extraglandular manifestations were similar among the three groups except that male patients showed lower frequency of arthritis and Raynaud's phenomenon compared with female patients (table 1).

No differences, were found among the immunological profile of children and female adult patients. The immunological profile of male patients showed a lower frequency of ANA $(p<0.025)$ and antibodies to $\operatorname{Ro}(S S A)$ nuclear antigens $(p<0.025)$ compared with female patients (table 1 ).

\section{Discussion}

The main purpose of this study was to clarify the clinical and serological findings in paediatric and male SS patients, using at present uniformly accepted European criteria. ${ }^{11}$ In most of the cases reported in the medical literature previously, there is no distinction between primary and secondary SS. ${ }^{7-10}$

In our study, which is a controlled comparative study, we have excluded three children: two of them had another underlying disease, and the third did not have positive minor salivary gland biopsy. One man also was excluded because the minor salivary gland biopsy showed granuloma, compatible with sarcoidosis. We found that children showed the same glandular and systemic manifestations during the course of the disease compared with adult female patients. However, RPGE was the most common initial clinical manifestation in the majority of the children $(77 \%)$ compared with male and adult female patients. These findings are in agreement with most of the investigators who reported that RPGE is the major clinical finding in children..$^{7-10}$

On the other hand in a study of 36 men and 69 women with primary SS, Molina et al found that men run the same risk as women of developing systemic manifestations, but few had increase concentrations of RF and antiRo(SSA) antibodies. ${ }^{5}$ In this study we found that SS in male patients had a tendency to be less severe with lower frequency of arthritis and Raynaud's phenomenon and with lower frequency of autoantibodies in their serum. These findings are partially in agreement with those reported by Molina, who found more extraglandular manifestations and less autoantibodies in their male patients. However, our results are in agreement also with those reported by others who found that extraglandular manifestations in SS are associated with the presence of many autoantibodies. $^{1}$

Our results are in contrast with those reported by Anaya et al who found that male patients have more systemic manifestations, with more autoantibodies in their serum. ${ }^{6}$ These differences could be explained by the different genetic background in the study population. Primary SS patients have been found to be associated with the HLA-class II antigens, especially HLA-DRW ${ }_{52}$ or HLA$\mathrm{DR}_{3}$, or both, whereas SS accompanying rheumatoid arthritis is linked with the HLA-DR ${ }_{4}{ }^{12}$ In 1988 we reported that primary SS patients were associated with the HLA-DR alloantigen. The prevalence of HLA-DR 3 was found in only $30 \%$ of our patients with no statistical significance compared with normal controls. ${ }^{13}$ Studying the natural course of 120 SS patients we have identified eight cases with lymphomas. Six were called low grade (immunocytomas) and two intermediate grade non-Hodgkin's lymphomas. These lymphomas tend to evolve very slowly and may regress spontaneously ${ }^{14}$ and differ from those reported previously by Talal and Bunim who showed that high grade lymphomas were more common in SS patients. ${ }^{15}$ These genetic variabilities may contribute to the clinical differences observed in our SS male patients.

In conclusion, it seems that primary SS in paediatric and male patients is rare. Recurrent parotid gland enlargement is the most common clinical finding at disease onset in children. Male patients seem to have less systemic manifestations and lower frequency of autoantibodies.

We are indebted to professor $\mathrm{H} \mathrm{M} \mathrm{Moutsopoulos} \mathrm{for} \mathrm{critically}$ reading our manuscript and we also thank Mrs E Horti for her excellent secretarial assistance. 
1 Talal N, Moutsopoulos HM, Kassan SS, eds. Sjögren's syndrome: clinical and immunological aspects. Berlin: Springer Verlag, 1987

2 Moutsopoulos HM. Sjogren's syndrome: autoimmune epitheliitis. Clin Immunol Immunopathol 1994;72:162-5. 3 Mikulicz J. Discussion at Verein fur wissenschaftliche Heilkunde zu königsberg. Berl Klin Wocchenschr 1888; 25:759.

4 Hara Y, Mikaska N, Nishido T, Ukuda M. Clinical study on nine male cases with Sjogren's syndrome. Ryumachi 1979; 19:410-4.

5 Molina R, Provost TT, Arnett FC, Bias WB, Hochberg MC, Wilson RW, Alexander EL. Primary Sjogren's syndrome in men. Clinical, serological and immunogenetic features. Am J Med 1986;80:23-31.

6 Anaya JM, Lin GT, D'souza E, Ogawa N, Luan X, Talal N. Primary Sjogren's syndrome in men. Ann Rheum Dis Primary Sjogren's

7 Chudwin DS, Daniels TE, Wara DW, Ammann AJ, Barrett DJ, Whitcher JP, et al. Spectrum of Sjogren's syndrome in children. J Pediatr 1981; 98:213-7.

8 Mizuno Y, Hara T, Hatae K, Hirano T, Ueda K, Nakamura $\mathrm{N}$, et al. Recurrent parotid gland enlargement as an initial manifestation of Sjogren's syndrome in children. Eur J Pediatr 1989;148:414-6.
9 Siamopoulou-Mavridou A, Drosos AA, Andonopoulos AP. Sjogren's syndrome in childhood: report of two cases. Eur J Pediatr 1989;148:523-4.

10 Anaya JM, Ogowa N, Talal N. Sjogren's syndrome in childhood. J Rheumatol 1995; 22:1152-8.

11 Vitali C, Bombardieri S, Moutsopoulos HM, Balestrieri G, Bencivelli W, Bernstein RM, et al. Preliminary criteria for the classification of Sjogren's syndrome. Arthritis Rheum 1993;36:340-7.

12 Moutsopoulos HM, Mann DL, Johnson AH, Chused TM. Genetic differences between primary and secondary sicca syndrome. N Engl J Med 1979;301:761-3.

13 Papasteriades CA, Skopouli FN, Drosos AA, Andonopoulos AP, Moutsopoulos HM. HLA-alloantigen associations in Greek patients with Sjogren's syndrome. J Autoimmun 1988;1:85-90.

14 Pavlidis NA, Drosos AA, Papadimitriou C, Talal N, Moutsopoulos HM. Lymphoma in Sjogren's syndrome. Med Pediatr Oncol 1992;20:279-83.

15 Talal N, Bunim JJ. The development of malignant lymphoma in the course of Sjogren's syndrome. Am J Med 1964;36:529-40. 